\title{
AN ASYMPTOTIC ANALYSIS OF INTRACELLULAR SIGNALING GRADIENTS ARISING FROM MULTIPLE SMALL COMPARTMENTS*
}

\author{
RONNY STRAUBE ${ }^{\dagger}$ AND MICHAEL J. WARD $\ddagger$
}

\begin{abstract}
Intracellular signaling gradients naturally arise through a local activation of a diffusible signaling molecule, e.g., by a localized kinase, and a subsequent deactivation at a distant cellular site, e.g., by a cytosolic phosphatase. Here, we consider a spherical cell containing a finite number of small spherical compartments where a signaling molecule becomes activated by a localized enzyme. For the activation rate two cases are considered: a saturated enzyme with a constant rate and an unsaturated enzyme with a linear rate. Using the method of matched asymptotic expansions, we derive approximate solutions of the steady-state diffusion equation with a linear deactivation rate to obtain the three-dimensional concentration profile of activated signaling molecules inside the cell. Depending on the diffusion length of the signaling molecule, the profiles decay either exponentially or algebraically, where the mode of decay is described by an associated Green's function. Our analysis provides simple expressions for the local concentration profile in the neighborhood of a signaling compartment, which can be used to estimate the amplitude and the extent of the gradients in the respective regimes. The global concentration profiles also depend on the cell size and the particular spatial arrangement of the compartments relative to each other and to the cell boundary.
\end{abstract}

Key words. cell signaling, cellular regulation, asymptotic matching, Green's function, Neumann function, singular perturbations

AMS subject classifications. 92C05, 92C15, 35B25

DOI. $10.1137 / 080733401$

1. Introduction. Intracellular signal transduction often occurs by means of diffusible proteins or ions. Typically, these signal transducers are locally produced to convey biological information to distant cellular sites where they are degraded. In the simplest case the target sites are reached by diffusive transport. If the diffusion length of the protein or ion is smaller than the size of the cell, stationary concentration gradients may arise which can activate secondary events in a threshold-based manner.

$\mathrm{Ca}^{2+}$ ions are an important second messenger in living cells. Upon agonist stimulation they are released through ion channels residing in the cell membrane or from intracellular storage compartments, thereby producing localized increases in the cytosolic $\mathrm{Ca}^{2+}$ concentration which can span several orders of magnitude. Free $\mathrm{Ca}^{2+}$ is rapidly bound to mobile buffer proteins, which contribute to the formation of steadystate calcium gradients in the vicinity of an open ion channel [16, 17, 21, 24].

Another kind of intracellular gradient arises through reversible modification cycles of a signaling molecule by an antagonistic enzyme pair such as a kinase/phosphatase or guanosine nucleotide exchange factor (GEF)/GTPase activating protein (GAP) pair. For example, a kinase located at the cell membrane can phosphorylate a signaling molecule by adding one or more phosphate groups to certain amino acid residues. In

\footnotetext{
* Received by the editors August 22, 2008; accepted for publication (in revised form) March 2, 2009; published electronically May 13, 2009.

http://www.siam.org/journals/siap/70-1/73340.html

$\dagger$ Department of Systems Biology, Max-Planck-Institute for Dynamics of Complex Technical Systems, 39106 Magdeburg, Germany (rstraube@mpi-magdeburg.mpg.de). This author's research was supported by the Ministry of Education of Saxony-Anhalt within the Research Center 'Dynamic Systems.'

${ }^{\ddagger}$ Department of Mathematics, University of British Columbia, Vancouver, BC, V6T 1Z2, Canada (ward@math.ubc.ca). This author's research was supported by NSERC Canada.
} 
this way the signaling molecule becomes activated while it diffuses into the cytoplasm. There it can activate further molecules or translocate to the nucleus to trigger a transcriptional response of the cell before it is dephosphorylated (deactivated) by an associated phosphatase.

GEF/GAP pairs act in a similar way upon signaling molecules called small guanosine triphosphatases (GTPases) such as Ran. Upon binding to guanosine triphosphate (GTP) the Ran molecule becomes activated as RanGTP. In this state it can bind to other molecules to convey biological information. The signal can be turned on and off in a cyclic manner as the GAP catalyses the hydrolyzation (inactivation) from RanGTP to RanGDP, while the GEF leads to a reactivation by exchanging GDP for GTP in a RanGDP molecule. Signaling gradients of this kind have been observed during cell division and in motile cells in a series of experiments $[13,10,18,6,3]$. Theoretical investigations can be found in $[5,12,15,28,20]$.

Theory predicts that gradients of a diffusible signaling molecule may arise if the members of the corresponding kinase/phosphatase or GEF/GAP pair are localized to different intracellular compartments [5, 12]. Recent modeling efforts were restricted to the spherically symmetric case where one signaling compartment is located at the origin of a spherical cell [12], while other authors treated the signaling compartment as a point source in an infinite space $[20,16,17,21]$. Numerical studies in a finite domain were done in [24] for localized calcium signals and in [15] for signaling from the cell surface.

In this paper, we extend previous analytical modeling efforts by considering a collection of signaling compartments in a finite domain. In this way, the steady-state signaling properties and their dependence on the cell size are explicitly taken into account. Cell size and shape were recently shown to be important parameters in determining the signaling status in the cytosol [15]. In addition, it is conceivable that signaling may simultaneously occur from a multitude of compartments or molecular structures. For example, during mitosis, microtubule nucleation is initiated not only from centrosomes [25], but also around chromosomes, where a long-range gradient of RanGTP-Importin $\beta$ complexes regulates mitotic spindle formation [6]. On the other hand, it is now possible experimentally to use fluorophore-based real-time imaging techniques such as fluorescence resonance energy transfer to detect molecular interactions at subcellular scales, which, in principle, allows one to monitor signaling events from multiple compartments.

From a mathematical viewpoint, our model consists of solving the modified Helmholtz equation $\triangle u-\alpha^{2} u=0$, where $\alpha^{2}>0$ is a constant, in a spherical domain that contains $N$ small spherical compartments centered at arbitrary positions inside the unit sphere. It is assumed that the compartments are well separated in the sense that the distance between the centers of any two small spheres as well as to the cell boundary is $O(1)$ relative to the small $O(\varepsilon)$ radius of each of the spheres. The outer boundary on the unit sphere is reflecting, whereas we allow for two different specific forms of a flux-type boundary condition to be specified on the boundary of each small spherical compartment. The method of matched asymptotic expansions is then used to analyze this problem in the limit $\varepsilon \rightarrow 0$ of small compartmental radii for three different distinguished limits of the constant $\alpha>0$ with respect to $\varepsilon$. Depending on the range of $\alpha$ relative to $\varepsilon$, the asymptotic solution is constructed in terms of the Neumann Green's function and its regular part for either the Laplacian or the modified Helmholtz operator in the unit sphere. Related problems, in the context of calculating the spectrum of the Laplacian in a three-dimensional domain containing small spherical inclusions, have been studied in [19] and [27] (see also [26] for related 
work in three spatial dimensions). Some related work in a two-dimensional spatial domain with a biophysical application is given in [23] and [4].

In section 2 we describe the model setup and introduce dimensionless quantities. As a result, we will obtain a boundary value problem (BVP) in a singularly perturbed domain that describes the stationary distribution of signaling molecules inside a spherical cell. In section 3 we identify three different scaling regimes for this BVP. Subsequently, asymptotic solutions are derived in each of the three regimes and compared with those from numerical simulations in section 4. As an application of our results, in section 5 we estimate the type of signaling gradient in two experimental systems. Finally, a discussion of our results is given in section 6 .

2. Model description. The cell is modeled as a sphere of radius $R$ that contains $N$ small spherical compartments located at arbitrary positions $\bar{x}_{j}$ within the cell (Figure 2.1). Each compartment may have a different radius $\varepsilon \rho_{j} R$ (with $\varepsilon>0$ and $\left.\rho_{j} \leq 1\right)$ such that the radius of the largest compartment is $\varepsilon R$. Note that $\varepsilon$ is a natural small parameter in this description. For example, if the cell radius is $R=10 \mu \mathrm{m}$ and signaling occurs from the cell nucleus, which typically has a radius of $R / 10=1 \mu \mathrm{m}$, $\varepsilon$ would be 0.1 . For smaller organelles such as endosomes or supramolecular structures, $\varepsilon$ would be even smaller.

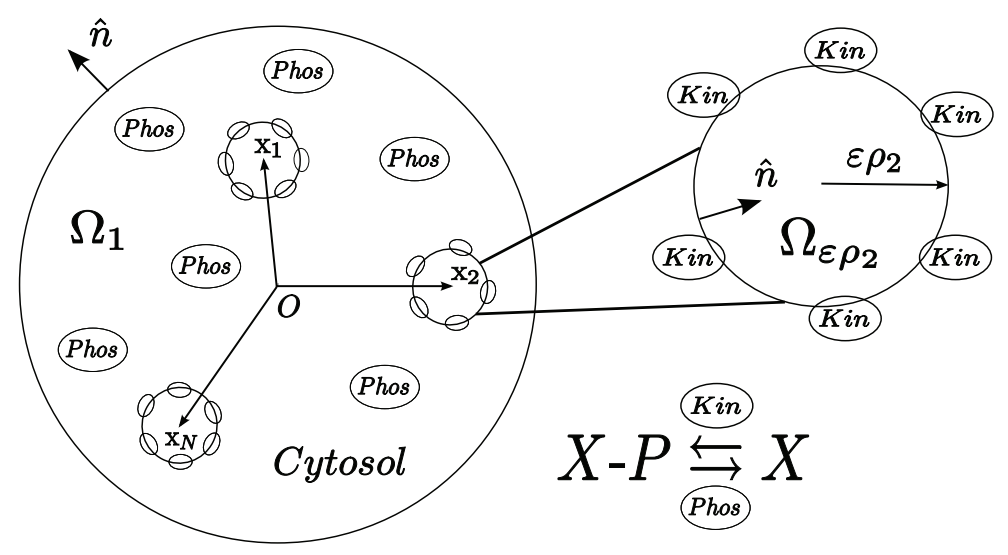

FIG. 2.1. Model setup in dimensionless units: Length scales are measured in terms of the cell radius $R$ as $x=\bar{x} / R$. The normal vector $\hat{n}$ points outward to the cytosolic region, i.e., inward to the internal compartments. The centers of these compartments are described by position vectors $x_{j}$ for $j=1, \ldots, N$. Spatial gradients of activated (phosphorylated) diffusible signaling molecules $X$ arise close to compartments $\Omega_{\varepsilon \rho_{j}}$, at the surface of which $X$ is converted into $X-P$ by a localized kinase (Kin). Far away from them $X$ is mainly found in the inactive (nonphosphorylated) form due to the action of a cytosolic phosphatase (Phos) on X-P.

We further assume that the activating enzyme (e.g., kinase or GEF) is exclusively localized to the intracellular compartments, whereas the antagonistic enzyme (e.g., phosphatase or GAP) is homogeneously distributed throughout the cytosol. The concentration of activated molecules is denoted as $c_{a}$. Once activated the molecule diffuses away from the site of activation into the cytosol where it is converted back to the inactive form. We take the deactivation rate $r_{d}$ proportional to the concentration of activated molecules; i.e., $r_{d}=k c_{a}$, where $k$ is the apparent first order rate constant of the cytosolic enzyme. For simplicity we take the diffusion coefficients of the active and inactive forms of the signaling molecule to be equal. With this assumption it is sufficient to consider the active form alone since the concentration of deactivated 
proteins can be expressed as $c_{d}=c_{t}-c_{a}$, where $c_{t}$ is the total abundance of signaling molecules in the cell. The stationary concentration profile for the active form is then determined by the BVP

$$
\begin{aligned}
D \Delta_{\bar{x}} c_{a}-k c_{a} & =0, \quad \bar{x} \in \Omega_{R} \backslash \Omega_{\varepsilon R}, \\
\partial_{n \bar{x}} c_{a} & =0, \quad \bar{x} \in \partial \Omega_{\varepsilon R}, \\
\varepsilon D \partial_{n \bar{x}} c_{a} & =r_{a, j}\left(c_{d}\right), \quad \bar{x} \in \partial \Omega_{\varepsilon \rho_{j} R},
\end{aligned}
$$

where $D$ is the diffusion coefficient of the activated signaling molecule, $\Delta_{\bar{x}}$ is the three-dimensional Laplace operator, and $\Omega_{\varepsilon R} \equiv \cup_{j=1}^{N} \Omega_{\varepsilon \rho_{j} R}$ is the union of all intracellular compartments, which when subtracted from $\Omega_{R}$ yields the cytosolic region. In addition, we impose a reflecting boundary condition at the cell surface in $(2.1 \mathrm{~b})$ and a flux boundary condition at the surface of each of the $N$ intracellular compartments in (2.1c), which models the local activation of the signaling molecules. Here, $\partial_{n \bar{x}} \equiv \hat{n} \cdot \nabla_{\bar{x}}$ denotes the outward normal derivative to the cytosolic region $\Omega_{R} \backslash \Omega_{\varepsilon R}$. Thus, $\hat{n}$ points inward to each of the small spheres (see Figure 2.1). The scaling in $\varepsilon \partial_{n \bar{x}}$ is chosen in such a way that, if $\bar{x}-\bar{x}_{j} \sim O(\varepsilon)$, the flux $r_{a, j}$ is of $O(1)$ near the $j$ th signaling complex.

2.1. Modeling the local activation rate. The substrate for the localized enzymes is the deactivated form of the signaling molecule $\left(c_{d}\right)$. For the activation rate we consider the two limiting cases of the Michaelis-Menten rate expression

$$
v_{a, j}=\frac{V_{a, j} c_{d}}{K_{a, j}+c_{d}} \approx \begin{cases}V_{a, j}, & c_{d} \gg K_{a, j}, \\ k_{a, j} c_{d}, & c_{d} \ll K_{a, j},\end{cases}
$$

which corresponds to a saturated and an unsaturated enzyme, respectively. Here, $k_{a, j} \equiv V_{a, j} / K_{a, j}$ is the apparent first order reaction rate for the activating enzyme. To obtain an expression for $r_{a, j}$ in (2.1c) we have to multiply $v_{a, j}$ by a length scale since the enzyme is assumed to be located at a surface. Consequently, $r_{a, j}$ has units $\mathrm{mol} \cdot \mathrm{m} / \mathrm{s}$.

We will now argue that a reasonable choice for the scaling factor is given by $\rho_{j} R / 3$ if the enzyme is saturated; i.e., $r_{a, j}=V_{a, j} \rho_{j} R / 3$ for $c_{d} \gg K_{a, j}$. To show this, we consider the local reaction-diffusion problem

$$
\Delta_{\bar{x}} c_{a}+\frac{I_{\varepsilon}}{\varepsilon^{2}} V_{a, j}=0, \quad \bar{x}-\bar{x}_{j}=O(\varepsilon),
$$

where $I_{\varepsilon}$ denotes the indicator function

$$
I_{\varepsilon}(\bar{x})= \begin{cases}1, & \left|\bar{x}-\bar{x}_{j}\right|<\varepsilon \rho_{j} R, \\ 0, & \left|\bar{x}-\bar{x}_{j}\right|>\varepsilon \rho_{j} R\end{cases}
$$

i.e., we assume that the activating enzyme is localized inside each compartment $\Omega_{\varepsilon \rho_{j} R}$ where the signaling molecule gets activated with a constant rate $V_{a, j}$. Subsequently, it diffuses into the cytosolic region where we locally solve the Laplace equation and require that $c_{a}$ vanish far away from the sphere. By introducing a local coordinate as $\bar{\rho} \equiv|\bar{y}|=\left|\bar{x}-\bar{x}_{j}\right| / \varepsilon$, we observe that the local activation rate is balanced by the diffusive transport and that the (radially symmetric) stationary concentration profile near the $j$ th compartment is determined by

$$
\begin{aligned}
\Delta_{\bar{\rho}} c_{a}+V_{a, j} & =0, & & 0<\bar{\rho}<\rho_{j} R, \\
\Delta_{\bar{\rho}} c_{a} & =0, & & \bar{\rho}>\rho_{j} R,
\end{aligned}
$$

Copyright (c) by SIAM. Unauthorized reproduction of this article is prohibited. 
where $\Delta_{\bar{\rho}} \equiv \partial_{\bar{\rho}}^{2}+(2 / \bar{\rho}) \partial_{\bar{\rho}}$ is the radial part of the Laplace operator. The solution of these equations readily follows from the requirement that $c_{a}$ be smooth across the boundary $\bar{\rho}=\rho_{j} R$. However, all we need here is an expression for the normal flux at the surface of the signaling compartment at $\bar{\rho}=\rho_{j} R$. Using the divergence theorem on the first equation, we obtain

$$
\int_{\Omega_{\rho_{j} R}} \Delta_{\bar{\rho}} c_{a} d \bar{y}=\left.4 \pi\left(\rho_{j} R\right)^{2} \partial_{\bar{\rho}} c_{a}\right|_{\rho_{j} R}=-\frac{4 \pi}{3} V_{a, j}\left(\rho_{j} R\right)^{3},
$$

which shows that

$$
-\partial_{\bar{\rho}} c_{a} \equiv \partial_{n \bar{y}} c_{a}=\varepsilon \partial_{n \bar{x}} c_{a}=V_{a, j} \frac{\rho_{j} R}{3}, \quad \bar{\rho}=\rho_{j} R .
$$

Thus, the particular choice for the $\varepsilon$-scaling in (2.1c) as well as the scaling factor $\rho_{j} R / 3$ is the result of the assumption that locally the diffusive transport is balanced by the enzymatic activation in (2.3). For simplicity, we will use the same scaling factor also for the unsaturated enzyme; i.e., we set

$$
r_{a, j}= \begin{cases}V_{a, j} \frac{\rho_{j} R}{3}, & \text { saturated enzyme, } \\ k_{a, j}\left(c_{t}-c_{a}\right) \frac{\rho_{j} R}{3}, & \text { unsaturated enzyme. }\end{cases}
$$

2.2. Dimensionless quantities. By introducing dimensionless quantities as $x=\bar{x} / R$ and $u=c_{a} / c_{t}$, the BVP (2.1) assumes the dimensionless form

$$
\begin{aligned}
\Delta_{x} u & =\alpha^{2} u, \quad x \in \Omega_{1} \backslash \Omega_{\varepsilon}, \\
\partial_{n x} u & =0, \quad x \in \partial \Omega_{1},
\end{aligned}
$$

where $\alpha=R / \lambda$ measures the diffusion length $\lambda=\sqrt{D / k}$ in terms of the cell radius, $\Omega_{\varepsilon} \equiv \cup_{j=1}^{N} \Omega_{\varepsilon \rho_{j}}$, and $\Omega_{1}$ is a sphere of radius one. In addition we have the local flux boundary conditions

$$
\varepsilon \partial_{n x} u=\left\{\begin{array}{ll}
\sigma_{j}, & (\mathrm{~A}) \\
\kappa_{j}(1-c), & (\mathrm{B})
\end{array} \quad x \in \partial \Omega_{\varepsilon \rho_{j}}, \quad j=1, \ldots, N .\right.
$$

The dimensionless parameters $\sigma_{j}$ and $\kappa_{j}$ are given by $\sigma_{j}=V_{a, j} \tau \rho_{j} / 3 c_{t}$ and $\kappa_{j}=$ $k_{a, j} \tau \rho_{j} / 3$, where $\tau \equiv R^{2} / D$ is the diffusion time. For later reference, we also define the kinetic parameters $\tilde{\sigma}_{j}$ and $\tilde{\kappa}_{j}$ as

$$
\tilde{\sigma}_{j} \equiv \frac{\sigma_{j}}{\rho_{j}}=\frac{V_{a, j} \tau}{3 c_{t}} \quad \text { and } \quad \tilde{\kappa}_{j} \equiv \frac{\kappa_{j}}{\rho_{j}}=\frac{k_{a, j} \tau}{3}
$$

which are independent of the radius $\rho_{j}$.

2.3. Physiological parameter values. In (2.6c) it is assumed that $\sigma_{j}$ and $\kappa_{j}$ are $O(1)$ quantities; i.e., $\sigma_{j} / \varepsilon$ and $\kappa_{j} / \varepsilon$ are $O(1 / \varepsilon) \gg 1$ depending on the size of the compartment. To estimate under what conditions this assumption is fulfilled we take a typical diffusion coefficient for a globular protein in the range $1-20 \mu \mathrm{m}^{2} / \mathrm{s}$ $[29,8,6]$, which fixes the diffusion time $\tau=R^{2} / D$ in the range $5-100$ s for a cell of radius $R=10 \mu \mathrm{m}$. As a conservative estimate for the activities $V_{a, j} / c_{t}$ and $k_{a, j}$ of the localized enzyme we can assume values in the range $1-10 / \mathrm{s}$, which shows that the flux $\partial_{n x} u$ can become as large as $O\left(10^{2}\right)$ corresponding to $\epsilon=O\left(10^{-2}\right)$. The biological significance of this estimate lies in the observation that, depending on the size of the compartment from which signaling occurs, the product of the diffusion time and the local enzyme activities must be sufficiently large $(\sim O(1 / \varepsilon))$ for a cell to locally produce an $O(1)$ flux of activated signaling molecules near each compartment. 
3. Asymptotic solutions. A straightforward solution of the BVP (2.6) is complicated by the fact that the domain $\Omega_{1}$ is perturbed by the presence of $N$ spherical holes inside $\Omega_{1}$. However, if $\varepsilon$ is sufficiently small, (2.6) becomes a BVP in a singularly perturbed domain for which powerful asymptotic methods, based on the method of matched asymptotic expansions, are available [11, 26, 7, 23, 19, 27]. The general idea is that the small holes act as point sources of a particular strength for the outer solution, which is obtained by solving the BVP (2.6) in the limit $\varepsilon \rightarrow 0$. The strength and the particular type of the singularity of the outer solution close to the $j$ th hole are obtained by asymptotically matching an appropriate inner solution (which has to fulfill either of the boundary conditions in (2.6c)) together with an outer solution, which is valid away from the localized compartments.

3.1. Different scaling regimes. By applying the divergence theorem to (2.6) and using either of the boundary conditions (2.6c), we may derive the following two exact relations from which the order of magnitude of the outer solution can be easily inferred. For BVP (A) we obtain

$$
\int_{\Omega_{1} \backslash \Omega_{\varepsilon}} u^{A}(x) d x=\frac{\varepsilon}{\alpha^{2}} \sum_{j=1}^{N} \sigma_{j}\left|\partial \Omega_{\rho_{j}}\right|,
$$

where $u^{A}$ denotes the solution of BVP (A) and $\left|\partial \Omega_{\rho_{j}}\right|=4 \pi \rho_{j}^{2}$ is the area of the rescaled spherical hole $\Omega_{\rho_{j}}$; i.e., $\left|\partial \Omega_{\rho_{j}}\right|=\varepsilon^{-2}\left|\partial \Omega_{\varepsilon \rho_{j}}\right|$. For BVP (B) the integration yields the relation

$$
\int_{\Omega_{1} \backslash \Omega_{\varepsilon}} u^{B}(x) d x=\frac{\varepsilon}{\alpha^{2}} \sum_{j=1}^{N} \kappa_{j}\left|\partial \Omega_{\rho_{j}}\right|\left(1-\bar{u}_{j}^{B}\right),
$$

where $\bar{u}_{j}^{B}$ is the average of $u^{B}$ over the surface of the $j$ th sphere. If $\alpha^{2}=O(1)$, then the right-hand sides in (3.1) and (3.2) are of $O(\varepsilon)$, which suggests that $u^{A}$ and $u^{B}$ are also of $O(\varepsilon)$ far away from the small holes. Consequently, we expect that the solutions decay rapidly far away from the signaling compartments, which results in large spatial gradients. Alternatively, if $\alpha^{2}=O(\varepsilon)$, then $u^{A}$ and $u^{B}$ are of $O(1)$ far away from the small holes allowing for long-range gradients. Note that if we had assumed in (2.6c) the boundary condition $\partial_{n x} u=\sigma_{j}$ with $\sigma_{j}=O(1)$ (i.e., without the $\varepsilon$ prefactor), we would have concluded from (3.1) that $u^{A}$ is of $O\left(\varepsilon^{2}\right)$ in most of the cell, i.e., that it was basically zero. In such a case the amplitude of the signal would only be of $O(\varepsilon)$ near each compartment.

A third scaling regime is motivated by the following observation: Near each hole we can introduce a rescaled coordinate $y=\left(x-x_{j}\right) / \varepsilon$ of $O(1)$ such that (2.6a) becomes

$$
\Delta_{y} u=\varepsilon^{2} \alpha^{2} u, \quad|y|=\rho \geq \rho_{j} .
$$

Now, if $\alpha^{2}=O(1)$ or $\alpha^{2}=O(\varepsilon)$, the dominant term of the inner solution will satisfy Laplace's equation, which has only algebraically decaying solutions. In contrast, if $\alpha=\alpha_{2} / \varepsilon$ with $\alpha_{2}=O(1)$, i.e., the diffusion length $\lambda$ becomes comparable with the radius $\varepsilon$ of the signaling compartment, the dominant term will solve the modified Helmholtz equation $\Delta_{y} u=\alpha_{2}^{2} u$, which allows for exponentially decaying solutions in the inner region. Consequently, this scaling regime yields highly localized solutions, which rapidly decay to zero outside the inner region while the local amplitude is still of $O(1)$. 
3.1.1. Scaling regimes and local activities. Before deriving explicit expressions for the asymptotic solutions of the BVP in (2.6) in the three different regimes identified above, we note that the order of magnitude of $\alpha$ also determines a reasonable choice for the local enzyme kinetics to some extent. For example, if the cytosolic degradation rate is weak $(\alpha=O(\sqrt{\varepsilon}))$, most of the signaling molecules will be in the activated state. Consequently, the concentration of inactivated proteins, which act as substrates for the localized enzyme, is very low such that it is likely to operate in the linear regime described by BVP (B) in (2.6c). Conversely, if $\alpha=O(1 / \varepsilon)$, the substrate concentration is so high that it is mostly saturating for the localized enzyme, resulting in the constant activation rate described by BVP (A) in (2.6c). It is only in the intermediate regime that both rate laws may reasonably be applied.

3.2. Solution for BVP (A) and $\alpha=\boldsymbol{O}(\mathbf{1})$. In the outer region of the $j$ th compartment, defined by $\left|x-x_{j}\right| \gg \varepsilon \rho_{j}$ for $j=1, \ldots, N$, we expand the solution of $\operatorname{BVP}(\mathrm{A})$ as

$$
u(x)=\varepsilon u_{1}(x)+\varepsilon^{2} u_{2}(x)+\cdots .
$$

Substituting this expansion into (2.6) leads to

$$
\begin{aligned}
\Delta_{x} u_{i}-\alpha^{2} u_{i}=0, & x \in \Omega_{1} \backslash\left\{x_{1}, \ldots, x_{N}\right\}, \\
\partial_{n x} u_{i}=0, & x \in \partial \Omega_{1}, \quad i=1,2 .
\end{aligned}
$$

Note that in the limit $\varepsilon \rightarrow 0$ each spherical hole $\Omega_{\varepsilon \rho_{j}}$ shrinks to the point $x_{j}$ at which it is centered. These points have to be excluded from the domain of definition for the outer problem (3.5). Instead, we shall derive singularity conditions for $u(x)$ as $x \rightarrow x_{j}$ by matching the outer solution to an appropriate inner solution defined near each $x_{j}$.

In the inner region, defined by $\left|x-x_{j}\right| \sim O(\varepsilon)$ for $j=1, \ldots, N$, we let $v_{j}(y)=$ $u\left(x_{j}+\varepsilon y\right)$, where $y=\varepsilon^{-1}\left(x-x_{j}\right)$ is a rescaled variable of $O(1)$ such that the radius of the $j$ th rescaled spherical hole is simply $\rho_{j}$. We then expand $v_{j}$ as

$$
v_{j}(y)=v_{j, 0}(y)+\varepsilon v_{j, 1}(y)+\varepsilon^{2} v_{j, 2}(y)+\cdots .
$$

For each $j=1, \ldots, N$ the rescaled BVP (A) (3.3) together with the boundary condition in $(2.6 \mathrm{c})$ becomes

$$
\begin{aligned}
\Delta_{y} v_{j} & =\varepsilon^{2} \alpha^{2} v_{j}, \quad|y| \equiv \rho \geq \rho_{j}, \\
\partial_{n y} v_{j} & =\sigma_{j}, \quad \rho=\rho_{j} .
\end{aligned}
$$

Upon substituting (3.6) into (3.7) and collecting powers of $\varepsilon$, we readily derive

$$
\begin{aligned}
\Delta_{y} v_{j, 0}=0, & \partial_{\rho} v_{j, 0}=-\sigma_{j}, \\
\Delta_{y} v_{j, 1}=0, & \partial_{\rho} v_{j, 1}=0, \\
\Delta_{y} v_{j, 2}=\alpha^{2} v_{0}, & \partial_{\rho} v_{j, 2}=0 .
\end{aligned}
$$

Note that since $\hat{n}$ points inward to each of the spherical holes, we have $\partial_{n y} \equiv-\partial_{\rho}$. The matching condition between the inner and outer expansions is that the nearfield behavior $\left(x \rightarrow x_{j}\right)$ of the outer solution must agree with the far-field behavior $\left(y=\left(x-x_{j}\right) / \varepsilon \rightarrow \infty\right)$ of the inner solution near the $j$ th hole; i.e.,

$$
\varepsilon u_{1}(x)+\varepsilon^{2} u_{2}(x)+\cdots=v_{j, 0}(y)+\varepsilon v_{j, 1}(y)+\varepsilon^{2} v_{j, 2}(y)+\cdots,
$$


to all orders in $\varepsilon$. To solve the inner BVP (3.8a) for $v_{j, 0}$, we impose that $v_{j, 0} \rightarrow 0$ as $\rho \rightarrow \infty$ and assume that $v_{j, 0}$ is radially symmetric so that $\Delta_{y} v_{j, 0}=0$ becomes $v_{j, 0}^{\prime \prime}+2 \rho^{-1} v_{j, 0}^{\prime}=0$. Then the solution is readily calculated as

$$
v_{j, 0}=\frac{\sigma_{j} \rho_{j}^{2}}{\rho} \equiv \frac{\sigma_{j}\left|\partial \Omega_{\rho_{j}}\right|}{4 \pi \rho}, \quad \rho \geq \rho_{j} .
$$

By rewriting this solution in terms of outer variables using $\rho=\left|x-x_{j}\right| / \varepsilon$, the first order matching condition becomes

$$
u_{1}(x) \sim \frac{\sigma_{j}\left|\partial \Omega_{\rho_{j}}\right|}{4 \pi\left|x-x_{j}\right|} \quad \text { as } \quad x \rightarrow x_{j}, \quad j=1, \ldots, N
$$

i.e., near the $j$ th spherical hole the first outer correction $u_{1}(x)$ is proportional to the free-space Green's function $G_{F}(x)=1 /\left(4 \pi\left|x-x_{j}\right|\right)$ of the Laplace equation, which satisfies $\Delta G_{F}(x)=-\delta\left(x-x_{j}\right)$. Consequently, we can extend the domain of definition of the outer BVP (3.5) for $u_{1}$ to all of $\Omega_{1}$ by including $\delta$-terms of strength $\sigma_{j}\left|\partial \Omega_{\rho_{j}}\right|$ for each singular point $x_{j}$. Then the first outer correction is determined from the BVP

$$
\begin{aligned}
\Delta_{x} u_{1}-\alpha^{2} u_{1} & =-\sum_{j=1}^{N} \sigma_{j}\left|\partial \Omega_{\rho_{j}}\right| \delta\left(x-x_{j}\right), \quad x \in \Omega_{1}, \\
\partial_{n x} u_{1} & =0, \quad x \in \partial \Omega_{1} .
\end{aligned}
$$

The solution of this equation is readily obtained in terms of the modified Helmholtz Green's function as

$$
u_{1}(x)=\sum_{j=1}^{N} \sigma_{j}\left|\partial \Omega_{\rho_{j}}\right| G_{h}\left(x ; x_{j}\right),
$$

where $G_{h}\left(x ; x_{0}\right)$ satisfies

$$
\Delta_{x} G_{h}-\alpha^{2} G_{h}=-\delta\left(x-x_{0}\right), \quad x \in \Omega_{1} ; \quad \partial_{n x} G_{h}=0, \quad x \in \partial \Omega_{1} .
$$

An explicit representation for this Green's function can be found by standard methods [2] with the result

$$
G_{h}\left(x ; x_{0}\right)=\frac{e^{-\alpha\left|x-x_{0}\right|}}{4 \pi\left|x-x_{0}\right|}+G_{R}\left(x ; x_{0}\right),
$$

where $G_{R}\left(x ; x_{0}\right)$ is defined by

$$
\begin{aligned}
G_{R}\left(x ; x_{0}\right) & =\sum_{n=0}^{\infty} \frac{2 n+1}{4 \pi \sqrt{r r_{0}}} P_{n}(\cos \gamma) \frac{a_{1, n}}{a_{2, n}} I_{n+1 / 2}(\alpha r) I_{n+1 / 2}\left(\alpha r_{0}\right), \\
\frac{a_{1, n}}{a_{2, n}} & =\frac{(\alpha R) K_{n+3 / 2}(\alpha R)-n K_{n+1 / 2}(\alpha R)}{(\alpha R) I_{n+3 / 2}(\alpha R)+n I_{n+1 / 2}(\alpha R)} .
\end{aligned}
$$

Here, $I_{\nu}(x)\left(K_{\nu}(x)\right)$ are modified Bessel functions of the first (second) kind of order $\nu$, while the argument of the Legendre polynomial $P_{n}(\cos \gamma)$ is given by $\cos \gamma=$ $\cos \theta \cos \theta_{0}+\sin \theta \sin \theta_{0} \cos \left(\varphi-\varphi_{0}\right)$ and $\left(r_{(0)}, \theta_{(0)}, \varphi_{(0)}\right)$ denotes the spherical coordinates of $x_{(0)}$. Due to our choice to work with dimensionless quantities, we simply 
have $R=1$ in (3.14). Note that the function $G_{R}\left(x ; x_{0}\right)$ is regular at the point $x=x_{0}$ and, therefore, can be expanded into a Taylor series as

$$
G_{R}\left(x ; x_{0}\right)=G_{R}\left(x_{0}, x_{0}\right)+\nabla_{x} G_{R}\left(x_{0}, x_{0}\right) \cdot\left(x-x_{0}\right)+\cdots .
$$

To proceed to the next order in the inner expansion (3.6) we need to know the far-field behavior of $v_{j, 1}$ at each hole as $\rho \rightarrow \infty$. For this reason, we expand $u_{1}(x)$ near the $j$ th hole using (3.11), (3.13), and (3.15) as

$$
\begin{aligned}
u_{1}(x)= & \sigma_{j}\left|\partial \Omega_{\rho_{j}}\right|\left(\frac{1}{4 \pi\left|x-x_{j}\right|}-\frac{\alpha}{4 \pi}+G_{R}^{j j}+\frac{\alpha^{2}}{8 \pi}\left|x-x_{j}\right|\right) \\
& +\nabla_{x} G_{R}^{j j} \cdot\left(x-x_{j}\right)+\sum_{\substack{i=1 \\
i \neq j}}^{N} \sigma_{i}\left|\partial \Omega_{\rho_{i}}\right|\left(G_{h}^{j i}+\nabla_{x} G_{h}^{j i} \cdot\left(x-x_{j}\right)\right)+\cdots
\end{aligned}
$$

Here, we have used the obvious notation $G_{(R)}^{j i} \equiv G_{(R)}\left(x_{j} ; x_{i}\right)$ as well as the fact that $G\left(x ; x_{i}\right)$ also has a well-defined Taylor expansion near $x_{j}$ as long as $i \neq j$. By rewriting this expansion in terms of the inner variable $y=\varepsilon^{-1}\left(x-x_{j}\right)$ with $|y|=\rho$, we obtain

$$
\varepsilon u_{1}=\frac{\sigma_{j}\left|\partial \Omega_{\rho_{j}}\right|}{4 \pi \rho}+\varepsilon\left(\sigma_{j}\left|\partial \Omega_{\rho_{j}}\right| G_{r e g}^{j j}+\sum_{\substack{i=1 \\ i \neq j}}^{N} \sigma_{i}\left|\partial \Omega_{\rho_{i}}\right| G_{h}^{j i}\right)+O\left(\varepsilon^{2}\right) .
$$

Upon comparing this expression with the matching condition (3.9), we find that the functions $v_{j, 1}$ asymptotically become constants as $|y| \rightarrow \infty$, which are given by

$$
v_{j, 1}(y) \sim \beta_{j}^{\sigma, \alpha} \equiv \sigma_{j}\left|\partial \Omega_{\rho_{j}}\right| G_{r e g}^{j j}+\sum_{\substack{i=1 \\ i \neq j}}^{N} \sigma_{i}\left|\partial \Omega_{\rho_{i}}\right| G_{h}^{j i}, \quad|y| \rightarrow \infty .
$$

Here, we have defined the regular part of the Green's function in (3.13) as

$$
G_{r e g}\left(x_{0} ; x_{0}\right)=-\frac{\alpha}{4 \pi}+G_{R}\left(x_{0} ; x_{0}\right) .
$$

Since the $v_{j, 1}$ are harmonic for $\rho \geq \rho_{j}$ and satisfy a no-flux boundary condition at $\rho=\rho_{j}$ (cf. (3.8b)) we obtain that $v_{j, 1} \equiv \beta_{j}^{\sigma, \alpha}$ for $\rho \geq \rho_{j}$ and $j=1, \ldots, N$; i.e., the $v_{j, 1}$ are constants everywhere outside each hole. As a consequence, the second order correction $u_{2}$ in the outer expansion (3.4) must satisfy the BVP (3.5), with no singularity as $x \rightarrow x_{j}$. The unique solution to this problem is simply $u_{2} \equiv 0$ everywhere in the outer region. In fact, one can show by solving (3.8c) for $v_{j, 2}$ together with its far-field behavior, which is determined by the $O\left(\varepsilon^{2}\right)$ terms in (3.17), that the next term in the outer expansion (3.4) is of $O\left(\varepsilon^{3}\right)$.

3.2.1. Local behavior of the solution. With the help of the local coordinate $\rho=\left|x-x_{j}\right| / \varepsilon$ as well as using (3.11) and (3.13), it is straightforward to show that the local behavior of the solution near the $j$ th hole is given by

$$
u(x)=\varepsilon \sigma_{j}\left|\partial \Omega_{\rho_{j}}\right| \frac{e^{-\alpha\left|x-x_{j}\right|}}{4 \pi\left|x-x_{j}\right|}+O(\varepsilon) \approx \sigma_{j} \rho_{j}^{2} \frac{e^{-\alpha \varepsilon \rho}}{\rho}, \quad \rho \geq \rho_{j} .
$$

The maximum value of the amplitude is attained at $\rho=\rho_{j}$. By writing $\sigma_{j}=\tilde{\sigma}_{j} \rho_{j}$ as in (2.7), where $\tilde{\sigma}_{j}$ is independent of $\rho_{j}$, we get

$$
u_{\max , j}^{\sigma} \approx \tilde{\sigma}_{j} \rho_{j}^{2} \exp \left(-\varepsilon \alpha \rho_{j}\right)
$$

Copyright ( $)$ by SIAM. Unauthorized reproduction of this article is prohibited. 
Thus, the maximal amplitude depends exponentially weakly on the cytosolic degradation rate $\alpha$, and it is proportional to the effective activation rate $\tilde{\sigma}_{j}$ and the surface area of the signaling compartment.

3.2.2. Limitations on the applicability of BVP (A). Due to the proportionality between $u_{\max , j}^{\sigma}$ and the effective activation rate $\tilde{\sigma}_{j}$ the former can become arbitrarily large as the latter increases. However, since we measure concentrations in terms of the total concentration of signaling molecules $c_{t}$, it must be required that $u_{\max , j}^{\sigma} \leq 1$, which restricts the applicability of this boundary condition to cases where

$$
\frac{V_{a, j}}{c_{t}}<\frac{3 e^{\varepsilon \alpha \rho_{j}}}{\rho_{j}^{2} \tau}, \quad j=1, \ldots, N
$$

i.e., the ratios between the source strength $V_{a, j}$ and the total concentration of signaling molecules $c_{t}$ must be sufficiently small.

3.3. Solution for BVP (B) and $\alpha=\boldsymbol{O}(1)$. In the outer region we, again, expand the solution as $u(x)=\varepsilon u_{1}(x)+\varepsilon^{2} u_{2}(x)+\cdots$. Substituting this expansion into the BVP (2.6) leads to the same set of outer problems as for BVP (A) (3.5). However, the inner problems will be different from the previous case due to the different boundary condition BVP (B) in (2.6c) at the surface of each compartment. Expanding the solution in the inner region as

$$
v_{j}(y)=v_{j, 0}(y)+\varepsilon v_{j, 1}(y)+\cdots,
$$

we readily derive the BVPs

$$
\begin{aligned}
& \Delta_{y} v_{j, 0}=0, \quad \partial_{\rho} v_{j, 0}=-\kappa_{j}\left(1-v_{j, 0}\right), \\
& \Delta_{y} v_{j, 1}=0, \quad \partial_{\rho} v_{j, 1}=\kappa_{j} v_{j, 1} .
\end{aligned}
$$

The radially symmetric solution of (3.23a), which decays at infinity, is given by

$$
v_{j, 0}=\frac{C_{j}\left|\partial \Omega_{\rho_{j}}\right|}{4 \pi \rho}, \quad C_{j} \equiv \frac{\kappa_{j}}{1+\rho_{j} \kappa_{j}} .
$$

Similarly as for BVP (A), the first outer correction is determined from

$$
\begin{aligned}
\Delta_{x} u_{1}-\alpha^{2} u_{1} & =-\sum_{j=1}^{N} C_{j}\left|\partial \Omega_{\rho_{j}}\right| \delta\left(x-x_{j}\right), \quad x \in \Omega_{1}, \\
\partial_{n x} u_{1} & =0, \quad x \in \partial \Omega_{1} .
\end{aligned}
$$

However, this is the same equation as (3.10) with $\sigma_{j}$ replaced with $C_{j}$. Thus, the solution is simply $u_{1}(x)=\sum_{j=1}^{N} C_{j}\left|\partial \Omega_{\rho_{j}}\right| G_{h}\left(x ; x_{j}\right)$, where $G_{h}\left(x ; x_{0}\right)$ is the modified Helmholtz Green's function given by (3.13).

By comparing the small argument expansion of $u_{1}$ near the $j$ th hole (cf. (3.16)) with the inner expansion (3.22), we observe that the far-field behavior of the functions $v_{j, 1}$ is given by

$$
v_{j, 1}(y) \sim \beta_{j}^{C, \alpha} \equiv C_{j}\left|\partial \Omega_{\rho_{j}}\right| G_{r e g}^{j j}+\sum_{\substack{i=1 \\ i \neq j}}^{N} C_{i}\left|\partial \Omega_{\rho_{i}}\right| G_{h}^{j i}, \quad|y| \rightarrow \infty .
$$

Copyright $@$ by SIAM. Unauthorized reproduction of this article is prohibited. 
TABLE 3.1

Asymptotic solutions of BVP (2.6) together with the corresponding boundary conditions denoted as $B V P(\mathrm{~A})$ and $B V P(\mathrm{~B})$ in $(2.6 \mathrm{c})$.

\begin{tabular}{|l|l|l|}
\hline$\alpha$ & Asymptotic solution of BVP $(2.6)$ & BVP \\
\hline$O(\sqrt{\varepsilon})$ & $u^{\sqrt{\varepsilon}}=u_{0}+\varepsilon\left(1-u_{0}\right) \sum_{j=1}^{N} C_{j}\left|\partial \Omega_{\rho_{j}}\right|\left(G_{n}\left(x ; x_{j}\right)-\frac{\beta_{j}^{C}}{C_{\alpha}}\right)+O\left(\varepsilon^{2}\right)$ & (B) \\
\hline$O(1)$ & $u^{\sigma}=\varepsilon \sum_{j=1}^{N} \sigma_{j}\left|\partial \Omega_{\rho_{j}}\right| G_{h}\left(x ; x_{j}\right)+O\left(\varepsilon^{3}\right)$ & (A) \\
\hline$O(1)$ & $u^{\kappa}=\varepsilon \sum_{j=1}^{N} C_{j}\left|\partial \Omega_{\rho_{j}}\right| G_{h}\left(x ; x_{j}\right)\left(1-\varepsilon \beta_{j}^{C, \alpha}\right)+O\left(\varepsilon^{3}\right)$ & (B) \\
\hline$O(1 / \varepsilon)$ & $u^{1 / \varepsilon}=\varepsilon \sum_{j=1}^{N} \frac{\sigma_{j}\left|\partial \Omega_{j}\right| e^{\alpha_{2} \rho_{j}}}{1+\alpha_{2} \rho_{j}} G_{f}\left(x ; x_{j}\right)$ & (A) \\
\hline
\end{tabular}

The (radially symmetric) solution of the BVP (3.23b) for $v_{j, 1}$ is then calculated as

$$
v_{j, 1}(\rho)=\beta_{j}^{C, \alpha}\left(1-\frac{C_{j}\left|\partial \Omega_{\rho_{j}}\right|}{4 \pi \rho}\right) .
$$

By rewriting $v_{j, 1}$ in terms of outer variables (using $\rho=\left|x-x_{j}\right| / \varepsilon$ ), we obtain from the matching condition (3.9) that

$$
u_{2} \sim-\frac{\beta_{j}^{C, \alpha} C_{j}\left|\partial \Omega_{\rho_{j}}\right|}{4 \pi\left|x-x_{j}\right|}, \quad x \rightarrow x_{j}
$$

Thus, $u_{2}$ is determined from the BVP

$$
\begin{aligned}
\Delta_{x} u_{2}-\alpha^{2} u_{2} & =\sum_{j=1}^{N} \beta_{j}^{C, \alpha} C_{j}\left|\partial \Omega_{\rho_{j}}\right| \delta\left(x-x_{j}\right), \quad x \in \Omega_{1}, \\
\partial_{n x} u_{2} & =0, \quad x \in \partial \Omega_{1},
\end{aligned}
$$

which has the solution $u_{2}(x)=-\sum_{j=1}^{N} \beta_{j}^{C, \alpha} C_{j}\left|\partial \Omega_{\rho_{j}}\right| G_{h}\left(x ; x_{j}\right)$.

In summary, we have shown that if $\alpha^{2}=O(1)$, then, up to terms of $O\left(\varepsilon^{3}\right)$, the solutions of BVP (A) and BVP (B) are given, respectively, by the expressions $u^{\sigma}$ and $u^{\kappa}$ shown in Table 3.1 .

3.3.1. Local behavior of the solution. Expanding $u^{\kappa}$ with the help of (3.13) near the $j$ th hole in the inner coordinate $\rho=\left|x-x_{j}\right| / \varepsilon$, we get

$$
u^{\kappa}(\rho)=C_{j} \rho_{j}^{2} \frac{e^{-\varepsilon \alpha \rho}}{\rho}\left(1-\varepsilon \beta_{j}^{C, \alpha}\right)+\cdots, \quad \rho \geq \rho_{j} .
$$

The comparison with numerical simulations in section 4 shows that the dominant term from the constants $\beta_{j}^{C, \alpha} \approx-C_{j} \rho_{j}^{2} \alpha$ (cf. (3.19) and (3.25)) has to be taken into consideration to correctly reproduce the maximal amplitude of $u^{\kappa}$ at $\rho=\rho_{j}$. By writing the constants $C_{j}$ in the form $C_{j}=\tilde{\kappa}_{j} \rho_{j} /\left(1+\tilde{\kappa}_{j} \rho_{j}^{2}\right)$, where $\tilde{\kappa}_{j} \equiv \kappa / \rho$ is independent of the radius $\rho_{j}$ (cf. (2.7)), the maximal amplitude can be approximated by

$$
u_{m a x, j}^{\kappa} \approx \frac{\tilde{\kappa}_{j} \rho_{j}^{2}}{1+\tilde{\kappa}_{j} \rho_{j}^{2}} e^{-\varepsilon \alpha \rho_{j}}\left(1+\varepsilon \frac{\tilde{\kappa}_{j} \rho_{j}^{2}}{1+\tilde{\kappa}_{j} \rho_{j}^{2}} \alpha \rho_{j}\right) .
$$

Note that $u_{m a x, j}^{\kappa}<1$ holds for any choice of the effective local enzyme activities $\tilde{\kappa}_{j}$.

Copyright $@$ ㅇ by SIAM. Unauthorized reproduction of this article is prohibited. 
3.4. Solution for BVP (B) and $\boldsymbol{\alpha}=\boldsymbol{O}(\sqrt{\varepsilon})$. We now assume that $\alpha^{2}=\varepsilon \alpha_{1}^{2}$ with $\alpha_{1}^{2}=O(1)$ and consider the BVP

$$
\begin{aligned}
\Delta_{x} u-\varepsilon \alpha_{1}^{2} u & =0, \quad x \in \Omega_{1} \backslash \Omega_{\epsilon}, \\
\partial_{n x} u & =0, \quad x \in \partial \Omega_{1}, \\
\varepsilon \partial_{n x} u & =\kappa_{j}(1-u), \quad x \in \partial \Omega_{\varepsilon \rho_{j}} .
\end{aligned}
$$

According to (3.2) we expect that in the outer region, as defined by $\left|x-x_{j}\right| \gg \varepsilon$, the solution of BVP (B) is of $O(1)$. This suggests an expansion of the form

$$
u(x)=u_{0}+\varepsilon u_{1}(x)+\varepsilon^{2} u_{2}(x)+\cdots .
$$

In the inner region near the $j$ th compartment we introduce the rescaled coordinate $y=\left(x-x_{j}\right) / \varepsilon$ with $\rho \equiv|y|$ and set $v_{j}(y)=u\left(x_{j}+\varepsilon y\right)$. The inner solution is then expanded as

$$
v_{j}(y)=u_{0}+v_{j, 0}(y)+\varepsilon v_{j, 1}(y)+\cdots,
$$

where the constant $u_{0}=O(1)$ will be found below. The matching condition reads

$$
u_{0}+\varepsilon u_{1}(x)+\varepsilon^{2} u_{2}(x)+\cdots=u_{0}+v_{j, 0}(y)+\varepsilon v_{j, 1}(y)+\cdots .
$$

Next, we insert the expansions (3.29) and (3.30) into (3.27), which yields the series of outer problems

$$
\begin{aligned}
\Delta_{x} u_{i} & =\alpha_{1}^{2} u_{i-1}, \quad x \in \Omega_{1} \backslash\left\{x_{1}, \ldots, x_{N}\right\}, \quad i=1,2, \\
\partial_{n x} u_{i} & =0, \quad x \in \partial \Omega_{1} .
\end{aligned}
$$

The corresponding inner problems are given by

$$
\begin{array}{ll}
\Delta_{y} v_{j, 0}=0, & \partial_{\rho} v_{j, 0}=-\kappa_{j}\left(1-u_{0}-v_{j, 0}\right), \\
\Delta_{y} v_{j, 1}=0, & \partial_{\rho} v_{j, 1}=\kappa_{j} v_{j, 1} .
\end{array}
$$

The inner solutions $v_{j, 0}$ are obtained from (3.32a) by imposing radial symmetry and the far-field condition $v_{j, 0} \rightarrow 0$ as $\rho \rightarrow \infty$ with the result

$$
v_{j, 0}(\rho)=\frac{\left(1-u_{0}\right) C_{j}\left|\partial \Omega_{\rho_{j}}\right|}{4 \pi \rho}, \quad C_{j}=\frac{\kappa_{j}}{1+\rho_{j} \kappa_{j}} .
$$

By rewriting this solution in terms of the outer variable using $\rho=\left|x-x_{j}\right| / \varepsilon$, the first order matching condition becomes

$$
u_{1}(x) \sim \frac{\left(1-u_{0}\right) C_{j}\left|\partial \Omega_{\rho_{j}}\right|}{4 \pi\left|x-x_{j}\right|}, \quad x \rightarrow x_{j}, \quad j=1, \ldots, N .
$$

This shows that the function $u_{1}$ is determined from

$$
\begin{aligned}
\Delta_{x} u_{1} & =\alpha_{1}^{2} u_{0}-\left(1-u_{0}\right) \sum_{j=1}^{N} C_{j}\left|\partial \Omega_{\rho_{j}}\right| \delta\left(x-x_{j}\right), \quad x \in \Omega_{1}, \\
\partial_{n x} u_{1} & =0, \quad x \in \partial \Omega_{1} .
\end{aligned}
$$

Copyright $@$ by SIAM. Unauthorized reproduction of this article is prohibited. 
Applying the divergence theorem to the first equation yields the solvability condition for this BVP which fixes the constant $u_{0}$ as

$$
u_{0}=\frac{\sum_{j=1}^{N} C_{j}\left|\partial \Omega_{\rho_{j}}\right|}{\alpha_{1}^{2}\left|\Omega_{1}\right|+\sum_{j=1}^{N} C_{j}\left|\partial \Omega_{\rho_{j}}\right|},
$$

where $\left|\Omega_{1}\right|=4 \pi / 3$ is the volume of the unit sphere. Using this expression for $u_{0}$ in (3.33), the solution $u_{1}$ is readily obtained as

$$
u_{1}(x)=\left(1-u_{0}\right) \sum_{j=1}^{N} C_{j}\left|\partial \Omega_{\rho_{j}}\right| G_{n}\left(x ; x_{j}\right)+\chi,
$$

where $\chi$ is a constant to be determined below. Here, $G_{n}\left(x ; x_{j}\right)$ is the Neumann function satisfying $\Delta_{x} G_{n}\left(x ; x_{0}\right)=1 /\left|\Omega_{1}\right|-\delta\left(x-x_{0}\right)$ for $x \in \Omega_{1}$ and $\partial_{n x} G_{n}=0$ for $x \in \partial \Omega_{1}$ together with the integral condition $\int_{\Omega_{1}} G_{n}\left(x ; x_{0}\right) d x=0$. For a sphere of radius $R$ it has the form [2]

$$
G_{n}\left(x ; x_{0}\right)=\frac{1}{4 \pi\left|x-x_{0}\right|}+R_{n}\left(x ; x_{0}\right),
$$

where the function $R_{n}\left(x ; x_{0}\right)$ is the regular part of this Green's function given by

$$
\begin{aligned}
R_{n}\left(x ; x_{0}\right)= & \frac{R}{4 \pi} \frac{1}{r\left|\frac{R^{2}}{r^{2}} x-x_{0}\right|}+\frac{1}{8 \pi R^{3}}\left(r^{2}+r_{0}^{2}\right)-\frac{7}{10 \pi R} \\
& +\frac{1}{4 \pi R} \log \left(\frac{2 R^{2}}{R^{2}-r r_{0} \cos \gamma+r\left|\frac{R^{2}}{r^{2}} x-x_{0}\right|}\right)
\end{aligned}
$$

with $\cos \gamma=\cos \theta \cos \theta_{0}+\sin \theta \sin \theta_{0} \cos \left(\varphi-\varphi_{0}\right)$ and where $\left(r_{(0)}, \theta_{(0)}, \varphi_{(0)}\right)$ are spherical coordinates of $x_{(0)}$. For our case of the unit sphere we set $R=1$ in (3.37).

The constant $\chi$ in (3.35) is obtained from the solvability condition for the second order outer problem in (3.31). However, to fully specify the BVP for $u_{2}$ we need to know its singular behavior as $x \rightarrow x_{j}$, which, in turn, is determined by the far-field behavior of the functions $v_{j, 1}$ of the inner expansion (3.30). For this reason, we expand $u_{1}(x)$ near the $j$ th hole as

$$
\begin{aligned}
\varepsilon u_{1}(x)= & \left(1-u_{0}\right) C_{j}\left|\partial \Omega_{\rho_{j}}\right|\left(\frac{\varepsilon}{4 \pi\left|x-x_{j}\right|}+\varepsilon R_{n}\left(x_{j} ; x_{j}\right)\right) \\
& +\varepsilon\left(1-u_{0}\right) \sum_{\substack{i=1 \\
i \neq j}}^{N} C_{i}\left|\partial \Omega_{\rho_{i}}\right| G_{n}\left(x_{j} ; x_{i}\right)+\varepsilon \chi+O\left(\varepsilon^{2}\right) \\
= & \frac{\left(1-u_{0}\right) C_{j}\left|\partial \Omega_{\rho_{j}}\right|}{4 \pi \rho}+\varepsilon\left(\left(1-u_{0}\right) \beta_{j}^{C}+\chi\right)+O\left(\varepsilon^{2}\right) .
\end{aligned}
$$

This has to be matched with the inner expansion $v_{j 0}(\rho)+\varepsilon v_{j 1}(\rho)+O\left(\varepsilon^{2}\right)$ showing that $v_{j, 1} \sim\left(1-u_{0}\right) \beta_{j}^{C}+\chi$ as $\rho \rightarrow \infty$, where $\beta_{j}^{C}$ is defined by

$$
\beta_{j}^{C} \equiv C_{j}\left|\partial \Omega_{\rho_{j}}\right| R_{n}\left(x_{j} ; x_{j}\right)+\sum_{\substack{i=1 \\ i \neq j}}^{N} C_{i}\left|\partial \Omega_{\rho_{i}}\right| G_{n}\left(x_{j} ; x_{i}\right) .
$$

Copyright (c) by SIAM. Unauthorized reproduction of this article is prohibited. 
The (radially symmetric) solution of the BVP for $v_{1},(3.32 \mathrm{~b})$, is simply

$$
v_{j, 1}(\rho)=\left(\left(1-u_{0}\right) \beta_{j}^{C}+\chi\right)\left(1-\frac{C_{j}\left|\partial \Omega_{\rho_{j}}\right|}{4 \pi \rho}\right), \quad \rho \geq \rho_{j} .
$$

This shows that $u_{2}$ is determined from the BVP

$$
\begin{aligned}
& \Delta_{x} u_{2}=\alpha_{1}^{2} u_{1}+\sum_{j=1}^{N}\left(\left(1-u_{0}\right) \beta_{j}^{C}+\chi\right) C_{j}\left|\partial \Omega_{\rho_{j}}\right| \delta\left(x-x_{j}\right), \quad x \in \Omega_{1}, \\
& \partial_{n x} u_{2}=0, \quad x \in \partial \Omega_{1} .
\end{aligned}
$$

Applying the divergence theorem to the first equation and using that $\int_{\Omega_{1}} u_{1} d x=$ $\left|\Omega_{1}\right| \chi$ yields the solvability condition

$$
\alpha_{1}^{2}\left|\Omega_{1}\right| \chi+\sum_{j=1}^{N}\left(\left(1-u_{0}\right) \beta_{j}^{C}+\chi\right) C_{j}\left|\partial \Omega_{\rho_{j}}\right|=0,
$$

from which the constant $\chi$ in (3.35) is obtained as

$$
\chi=-\left(1-u_{0}\right) \frac{\sum_{j=1}^{N} \beta_{j}^{C} C_{j}\left|\partial \Omega_{\rho_{j}}\right|}{\alpha_{1}^{2}\left|\Omega_{1}\right|+\sum_{j=1}^{N} C_{j}\left|\partial \Omega_{\rho_{j}}\right|} .
$$

In summary, if $\alpha^{2}=\varepsilon \alpha_{1}^{2}$, the asymptotic solution of BVP (B) is given by the expression for $u^{\sqrt{\varepsilon}}$ shown in Table 3.1, where $C_{\alpha} \equiv \alpha_{1}^{2}\left|\Omega_{1}\right|+\sum_{i=1}^{N} C_{i}\left|\partial \Omega_{\rho_{i}}\right|$ and the constants $u_{0}$ and $\beta_{j}^{C}$ are given in (3.34) and (3.38), respectively.

3.4.1. Local behavior of the solution. If we define $C_{N} \equiv 3 \sum_{i=1}^{N} C_{i} \rho_{i}^{2}$ and expand $u^{\sqrt{\varepsilon}}$ near the $j$ th signaling compartment using the inner coordinate $\rho=\left|x-x_{j}\right| / \varepsilon$ and (3.36), we get

$$
u^{\sqrt{\varepsilon}}(\rho)=\frac{C_{N}}{\alpha_{1}^{2}+C_{N}}+\frac{C_{j} \rho_{j}^{2} \alpha_{1}^{2}}{\alpha_{1}^{2}+C_{N}} \frac{1}{\rho}+O(\varepsilon), \quad \rho \geq \rho_{j} ;
$$

i.e., the algebraic gradient builds on a local background level which is determined by the sum of signaling strengths $\gamma_{i} \equiv C_{i} \rho_{i}^{2}$ of all other compartments. Note that $u^{\sqrt{\varepsilon}} \rightarrow 1$ as either $\alpha_{1} \rightarrow 0$ or $C_{N} \gg \alpha_{1}$.

3.5. Solution for BVP (A) and $\boldsymbol{\alpha}=\boldsymbol{O}(\mathbf{1} / \varepsilon)$. We set $\alpha=\alpha_{2} / \varepsilon$ in (2.6) with $\alpha_{2}=O(1)$. In that case the outer solution vanishes identically, and the rescaled BVP (3.3) becomes $\Delta_{y} v_{j}=\alpha_{2}^{2} v_{j}$ in contrast to (3.7). Here $y=\left(x-x_{j}\right) / \varepsilon$ denotes, again, the rescaled variable. In addition, the functions $v_{j}$ have to satisfy the boundary conditions $\partial_{n y} v_{j}=\sigma_{j}$ at the surface of each compartment. We expand the inner solution as $v_{j}(y)=v_{j, 0}(y)+\varepsilon v_{j, 1}(y)+\cdots$, which yields

$$
\begin{gathered}
\Delta_{y} v_{j, i}=\alpha_{2}^{2} v_{j, i}, \quad|y|=\rho \geq \rho_{j}, \quad i=0,1, \\
\partial_{\rho} v_{j, 0}=-\sigma_{j} \quad \text { and } \quad \partial_{\rho} v_{j, 1}=0, \quad \rho=\rho_{j} .
\end{gathered}
$$

The solutions of these BVPs are easily found if we impose radial symmetry and require that $v_{j, i} \rightarrow 0$ as $\rho \rightarrow \infty$ for $i=0,1$ and $j=1, \ldots, N$. As a result, the inner solution 
reads

$$
\begin{aligned}
& v_{j}(\rho)=\frac{\sigma_{j}\left|\partial \Omega_{j}\right| e^{\alpha_{2} \rho_{j}}}{1+\alpha_{2} \rho_{j}} \frac{e^{-\alpha_{2} \rho}}{4 \pi \rho} \text { or } \\
& v_{j}(x)=\varepsilon \frac{\sigma_{j}\left|\partial \Omega_{j}\right| e^{\alpha_{2} \rho_{j}}}{1+\alpha_{2} \rho_{j}} \frac{e^{-\alpha_{2}\left|x-x_{j}\right| / \varepsilon}}{4 \pi\left|x-x_{j}\right|}
\end{aligned}
$$

since $v_{j, 1}$ (and all higher corrections) vanish identically. The total concentration profile, as shown in Table 3.1, is a superposition of the form $u^{1 / \varepsilon}(x)=\sum_{j=1}^{N} v_{j}(x)$. The maximal amplitude at the $j$ th compartment is given by

$$
u_{\max , j}^{1 / \varepsilon} \approx \frac{\tilde{\sigma}_{j} \rho_{j}^{2}}{1+\alpha_{2} \rho_{j}},
$$

where $\tilde{\sigma}_{j} \equiv V_{a, j} \tau / 3 c_{t}$ is independent of $\rho_{j}$ (cf. (2.7)). Here, the same restrictions for the choice of $\tilde{\sigma}_{j}$ apply as those mentioned in section 3.2.2, i.e., $\tilde{\sigma}_{j}<\left(1+\alpha_{2} \rho_{j}\right) / \rho_{j}^{2}$.

4. Numerical simulations. Strictly speaking, the asymptotic solutions shown in Table 3.1 are valid only for sufficiently small $\varepsilon$. However, when comparing with numerical simulations, we found that they provide excellent approximations even for $\varepsilon=0.1$, which corresponds to the radius of a typical nucleus. BVP (2.6) was solved numerically using the COMSOL software package [1].

There is one basic restriction which limits the number of signaling compartments that can be modeled within the cell: The spatial arrangement of the compartments must be such that they influence each other only through their far-field; i.e., they must not be too close to each other or to the cell boundary. Otherwise higher orders in $\varepsilon$ have to be taken into account. This restriction is a direct consequence of our assumption that the near-field around each compartment is not disturbed by the presence of other compartments. Hence, our analysis does not treat the case of a spatial clustering of small compartments. Figure 4.1 shows the behavior of the asymptotic solutions in comparison with the numerical solutions as $\alpha$ changes from $O(\sqrt{\varepsilon})$ to $O(1)$ in (a)-(c) and from $\alpha=O(1)$ to $O(1 / \varepsilon)$ in (d)-(f) for one signaling compartment with radius $\varepsilon=0.1$. From these graphs it is apparent that each of the asymptotic solutions has a well-defined domain of validity. For example, at $\alpha=0.6$ (Figure 4.1(a)) the $O(1)$ solution $u^{\kappa}$ (solid line) underestimates the true concentration profile, while $u^{\sqrt{\varepsilon}}$ (dashed line) is in excellent agreement with the numerical solution (triangles). At the transition point $\alpha=1$ both solutions are equally good (Figure 4.1(b)), whereas at $\alpha=5$ the $O(1)$ solution $u^{\kappa}$ coincides with the result from numerical simulation, while $u^{\sqrt{\varepsilon}}$ begins to diverge. A similar scenario is observed during the transition from $\alpha=O(1)$ to $\alpha=O(1 / \varepsilon)$ (Figure 4.1(d)-(f)).

The restriction that the compartments not be too close to each other (or to the boundary) is certainly most limiting in the case of long-range signaling gradients $(\alpha=O(\sqrt{\varepsilon}))$ since they do not exhibit an intrinsic length scale. In particular, it is not a priori obvious how large the distance between compartments or from any compartment to the boundary has to be chosen in order for the asymptotic solution to be a reasonable approximation. In Figure 4.2 we compare the numerical solution with the asymptotic result $u^{\sqrt{\varepsilon}}$ in Table 3.1 for a configuration of three signaling compartments along the $x$-axis and $\alpha=0.7$. In Figure 4.2(a) the radius of the compartments is $\varepsilon=0.05$. The shortest distance to the cell boundary (as measured from the surface of the compartments) is $7 \varepsilon=0.35$, while the shortest distance between 

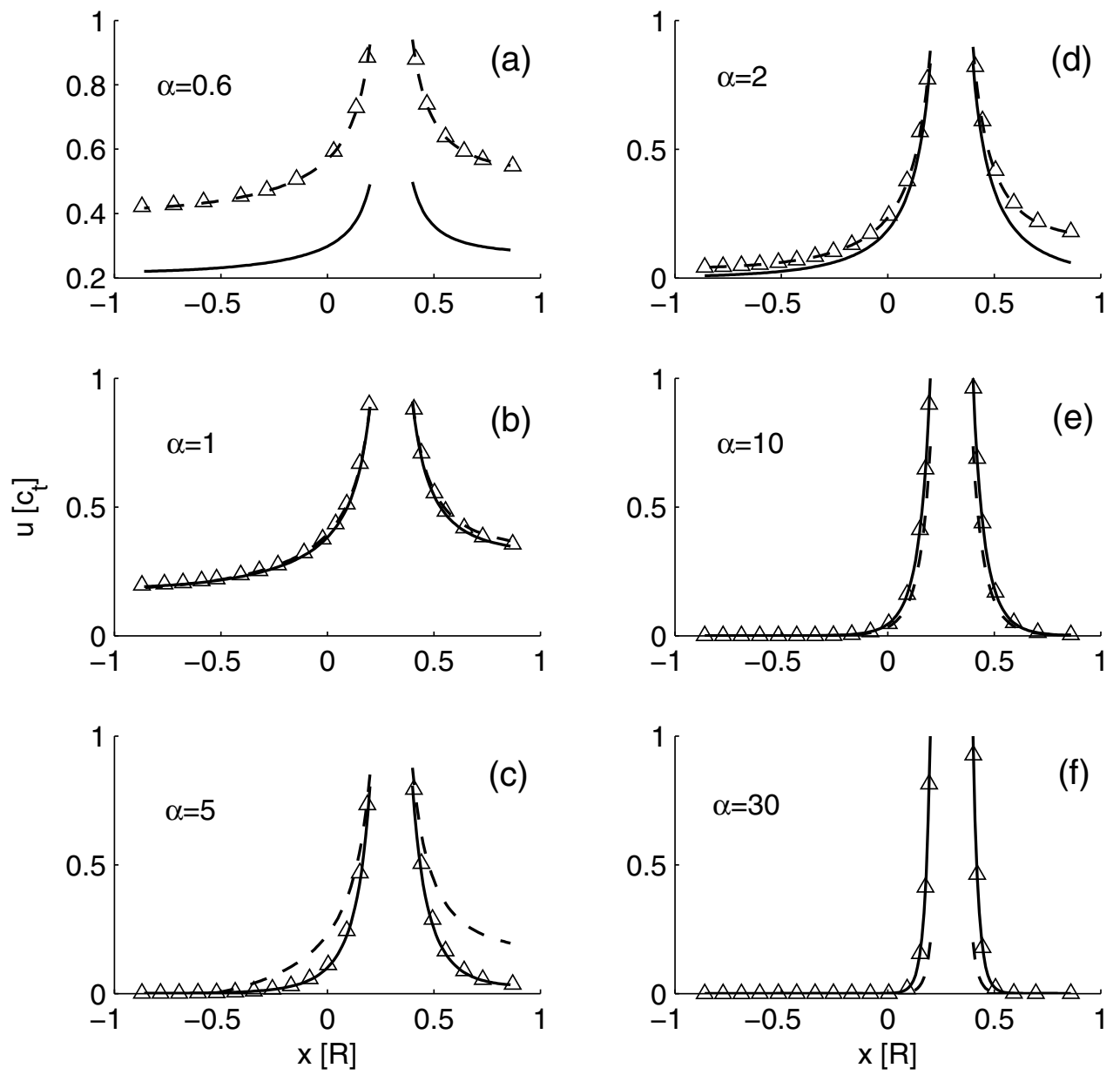

FIG. 4.1. Comparison of the asymptotic solutions shown in Table 3.1 with those from numerical simulations (triangles). (a)-(c) Transition from $\alpha=O(\sqrt{\varepsilon})$ to $O(1): u^{\sqrt{\varepsilon}}$ (dashed line) vs. $u^{\kappa}$ (solid line) with $\kappa=10$. (d)-(f) Transition from $\alpha=O(1)$ to $O(1 / \varepsilon): u^{\sigma}$ (dashed line) vs. $u^{1 / \varepsilon}$ (solid line) with $\sigma=(1,2,4)$. Radius and location of the compartment are $\varepsilon=0.1$ and $\left(x_{0}, y_{0}, z_{0}\right)=$ $(0.3,0.3,0.4)$, respectively.

compartments is $8 \varepsilon=0.4$. For this asymmetric configuration the asymptotic solution (marked by dots) nicely reproduces the numerical result (solid line). In Figure 4.2(b) the radius of the compartments has been increased to $\varepsilon=0.1$ such that the shortest distance to the cell boundary as well as between compartments is reduced to $3 \varepsilon=0.3$. However, the asymptotic solution still provides a very good approximation to the numerical solution, although the minima between each two compartments and at the left boundary are slightly underestimated. To appreciate the significance of the constant terms $\sim \beta_{j}^{C}$ in $u^{\sqrt{\varepsilon}}$ we have also plotted the solution without these contributions (dashed line) in Figure 4.2(b). Note that the whole correction term $\sim C_{j} \beta_{j}^{C}$ is quadratic in the $C_{j}$ and thus describes "interactions" between the compartments. A similar term also appears in the asymptotic solution $u^{\kappa}$ for $\alpha=O(1)$. However, here its contribution is only $O\left(\varepsilon^{2}\right)$. 

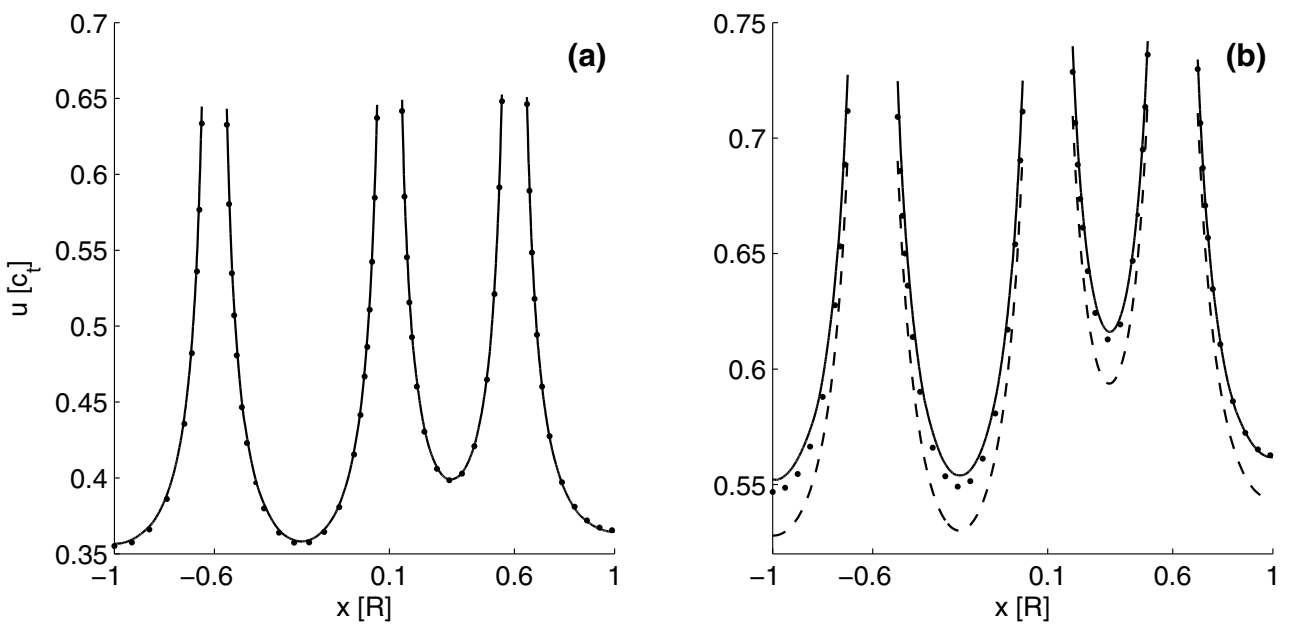

FIG. 4.2. Concentration profiles in the long-range regime $(\alpha=0.7)$ resulting from three signaling compartments located along the $x$-axis at $x_{1}=-0.6, x_{2}=0.1$, and $x_{3}=0.6$. Numerical solution: solid line; asymptotic solution $u^{\sqrt{\varepsilon}}$ in Table 3.1: black dots. $\kappa_{i}=\rho_{i}=1$ for $i=1,2,3$. (a) $\varepsilon=0.05$, (b) $\varepsilon=0.1$. The dashed line denotes the asymptotic solution $u^{\sqrt{\varepsilon}}$ without the "interaction terms" $\sim \beta_{j}^{C}$.

5. Comparison with experiments. In the following, we apply our theory to recent experiments which revealed the significance of intracellular signaling gradients for the regulation of mitotic spindle formation during cell division in eukaryotic cells. Here, our focus is on the estimation of the type of the signaling gradient, i.e., its dimensionless decay length $\alpha$. No attempt is made to reproduce the experimentally observed local amplitudes of the gradients. Consequently, we choose the local enzyme activities conveniently.

5.1. Phosphorylation gradient of stathmin-oncoprotein 18. Recent experiments in Xenopus A6 epithelial cells show that during cell division a phosphorylation gradient of stathmin-oncoprotein 18 (OP18) around mitotic chromatin is necessary for the mitotic spindle to form correctly [18]. Here, the nonphosphorylated form of OP18 has an inhibitory effect on microtubule growth through binding to tubulin dimers [9]. Upon phosphorylation by a chromatin-localized kinase (e.g., Polo kinase) the inhibitory effect of OP18 ceases, which locally promotes mitotic spindle assembly. OP18 is then dephosphorylated by a cytosolic phosphatase (e.g., protein phosphatase 2A). To estimate the type of signaling gradient, we use the parameter values $R=10 \mu \mathrm{m}, D=13-18 \mu \mathrm{m}^{2} / \mathrm{s}, k=0.3, \ldots, 0.7 \mathrm{~s}^{-1}$ from [18] and assume that the effective radius of the chromatin has the same order of magnitude as that of a nucleus; i.e., we set $\varepsilon=0.1$, which yields values for $\alpha$ in the range $\alpha=1.3, \ldots, 2.3$. The corresponding concentration profiles of OP18-P for $\alpha=1.3$ (bold solid line) and $\alpha=2.3$ (solid line) are shown in Figure 5.1(a). A 10-fold increase in the cytosolic phosphatase concentration does not lead to a qualitative change in the concentration profile, since the corresponding value for $\alpha$ is still below the threshold value for localized profiles $\alpha_{c}=1 / \varepsilon=10$ (Figure 5.1(a), dashed line). However, a 2-fold decrease yields $\alpha \approx 0.92$. In that case, the OP18-P profile already exhibits the typical algebraic decay which has no associated natural length scale (Figure 5.1(a), bold dashed line). This indicates that the OP18 signaling system operates close to the transition from 

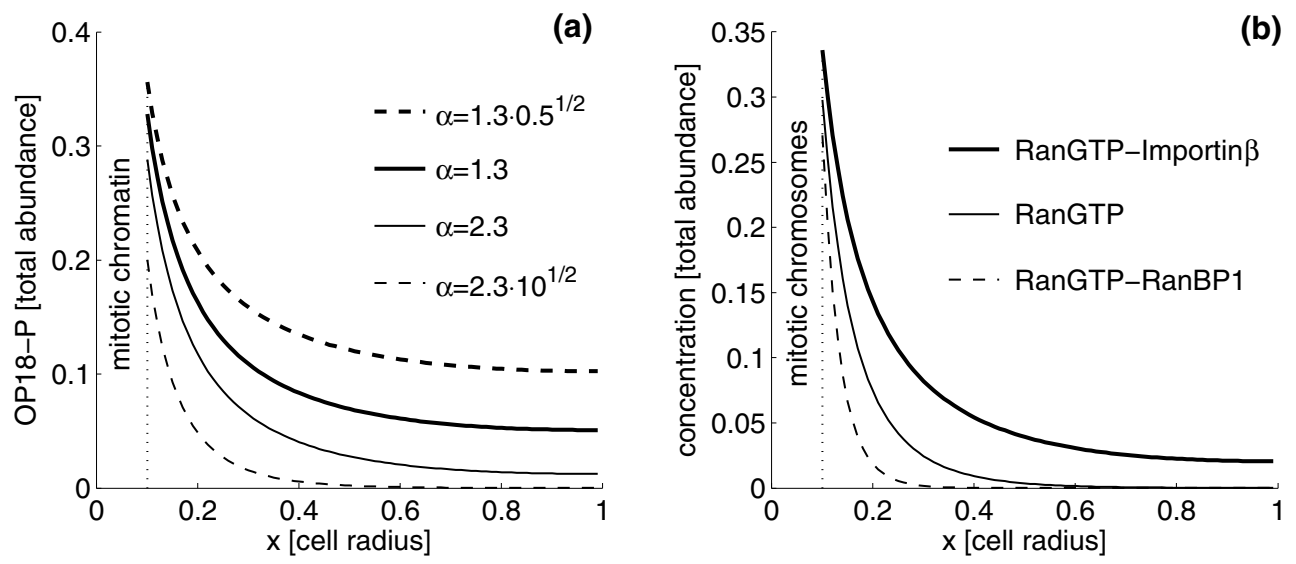

FIG. 5.1. Estimating the type of signaling gradient in two experimental systems. The signaling compartment (radius: $\varepsilon=0.1$ ) is localized at the origin. (a) Spatial distribution of phosphorylated OP18 protein for parameter values from [18]: $\alpha=1.3$ (bold solid line), $\alpha=2.3$ (solid line), a 2-fold decrease $\alpha=(0.5)^{1 / 2} \cdot 1.3 \approx 0.92$ (bold dashed line), and a 10 -fold increase $\alpha=(10)^{1 / 2} \cdot 2.3 \approx 7.3$ (dashed line) in cytosolic enzyme concentration. The effective activity of the chromatin localized kinase was set to $\kappa=0.5$. (b) Concentration profiles of different RanGTP species around mitotic chromosomes. The activation rate $\sigma$ was chosen (arbitrarily) as follows: RanGTP-Importin $\beta$ ( $\sigma=$ $0.4), \operatorname{Ran} G T P(\sigma=0.6)$, and RanGTP-RanBP1 $(\sigma=2)$.

the algebraic to the exponential decay regime. However, it is unclear whether and in which way the cell exploits this opportunity to selectively change the type of signaling gradient, e.g., by a corresponding up- or downregulation of the cytosolic enzyme concentration.

5.2. Different Ran-GTP gradients around mitotic chromosomes. During cell division microtubules grow from the two centrosomes and attach at the kinetochores of the sister chromatids which are located at the equatorial plane of the cell. Recent experiments demonstrate that the nucleation and stabilization of microtubules is selectively facilitated by different RanGTP gradients around chromosomes of mitotic cells [6]. The gradients are generated by the combined action of a chromatin-localized GEF (RCC1) and cytosolic RanGAP. A detailed model for the reactions occurring in the chromosomal and cytosolic compartments can be found in the supplemental material of [6]. Here, we aim only to estimate the effective cytosolic degradation rate $\alpha$ which determines the type of signaling gradient.

For definiteness we consider a cell of radius $10 \mu \mathrm{m}$ and an effective chromosome radius of $1 \mu \mathrm{m}$, i.e., $\varepsilon=0.1$. The diffusion coefficients for the different RanGTP species are taken from [6] as $20 \mu \mathrm{m}^{2} / \mathrm{s}$ (RanGTP), $17.2 \mu \mathrm{m}^{2} / \mathrm{s}$ (RanGTP-RanBP1), and $13 \mu \mathrm{m}^{2} / \mathrm{s}$ (RanGTP-Importin $\beta$ ). There are three distinct pathways through which RanGTP can be hydrolyzed to RanGDP (see Figures S3 and S4 in [6]). It can be directly hydrolyzed by the cytosolic RanGAP with an activity of $k=10 \mathrm{~s}^{-1}$, or it first associates with RanBP1 in a RanGTP-RanBP1 complex $\left(K_{d} \approx 1 \mathrm{nM}\right)$ which is then hydrolyzed with a 7-fold higher activity of $k=70 \mathrm{~s}^{-1}$. Finally, RanGTP can bind to the karyopherin Importin $\beta\left(K_{d} \approx 1 \mathrm{nM}\right)$, which prevents its hydrolysis to RanGDP by RanGAP. However, the association of RanBP1 with the RanGTPImportin $\beta$ complex induces the immediate release of RanGTP from Importin $\beta$. In [6] the association rate has been taken as $k_{o n}=0.3 \mu \mathrm{M}^{-1} \mathrm{~s}^{-1}$, and the cytosolic RanBP1 concentration was assumed to be $2 \mu \mathrm{M}$, which yields an apparent degradation rate for 
the RanGTP-Importin $\beta$ complex of $k=0.6 \mathrm{~s}^{-1}$. This shows that the gradient for the RanGTP-Importin $\beta$ complex $(\alpha=2)$ is close to the transition between algebraic and exponential decay (Figure 5.1(b)). On the other hand, the concentration profile for the RanGTP-RanBP1 complex $(\alpha=20)$ is highly localized to the mitotic chromosomes, whereas the RanGTP gradient $(\alpha=7)$ is of the intermediate type.

6. Discussion. We have investigated intracellular signaling gradients as they arise through local activation of a diffusible signaling molecule by a collection of localized enzymes followed by global deactivation in the cytosol of a spherical cell. Using asymptotic methods, we derived explicit expressions for the stationary concentration profile of activated signaling molecules for small $(\alpha=O(\sqrt{\varepsilon}))$, intermediate $(\alpha=O(1))$, and large $(\alpha=O(1 / \varepsilon))$ values of the effective cytosolic enzyme activity $\alpha$ in combination with a constant (BVP (A)) and/or linear (BVP (B)) activation rate for the localized enzymes (Table 3.1). We find excellent agreement between the asymptotic results and numerical simulations up to $\varepsilon=0.1$ (Figures 4.1 and 4.2). The decay behavior of the profiles is determined by the dimensionless quantity $\alpha=R / \lambda$, which measures the diffusion length $\lambda=\sqrt{D / k}$ in terms of the cell radius $R$. Our analysis of the BVP (2.6) shows that there are three types of signaling gradients (cf. Table 3.1 and Figure 6.1). In each regime the mode of decay is described by an associated Green's function, which also sets the length scale for the corresponding gradient (Table 6.1).
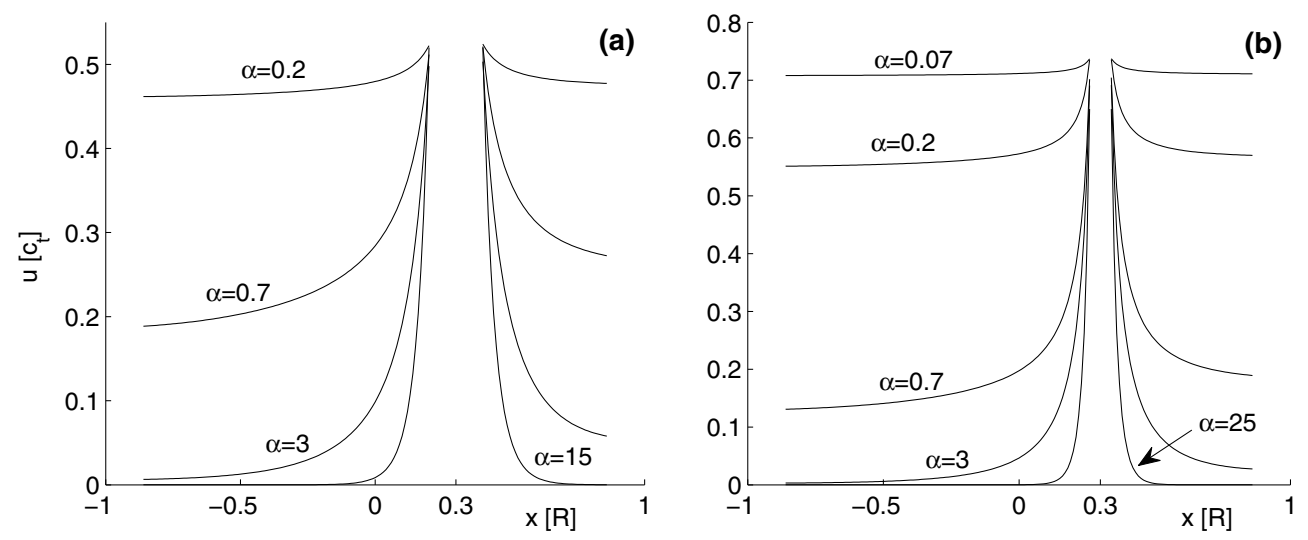

FIG. 6.1. Transition from $\alpha=O(\sqrt{\varepsilon})$ to $\alpha=O(1 / \varepsilon)$ for different compartment sizes: $\varepsilon=0.1$ (a) and $\varepsilon=0.04$ (b). Local activities are (from top to bottom) $\kappa=0.13, \kappa=0.7, \kappa=1.3, \sigma=1.3$ (a) and $\kappa=0.11, \kappa=0.7, \kappa=2, \kappa=2.5, \sigma=1.3(\mathrm{~b})$. Note that in (b) the profile still decays algebraically at $\alpha=0.2$, while in (a) it is basically constant.

It is interesting to note that the transition point between the three decay regimes is to a certain extent determined by the size of the compartment from which signaling occurs (Figure 6.1). For example, if the diffusion length is in the order of magnitude of the size of a compartment, i.e., $\lambda=O(\varepsilon R)$ or $\alpha=O(1 / \varepsilon)$, the activated signaling molecule will typically explore only a narrow region around the compartment before it gets deactivated by the cytosolic enzyme. This, in turn, leads to a highly localized (exponentially decaying) concentration profile as described by the (free space) Green's function $G_{f}$ in Table 6.1 , because in that regime the cell boundary has no influence on the local concentration profile. As the diffusion length grows, the mode of decay remains exponential until $\lambda$ becomes comparable in magnitude to the cell radius, i.e., 
TABLE 6.1

Green's functions and associated length scales.

\begin{tabular}{llc}
\hline$\alpha$ & Green's functions & Length scale \\
\hline$O(\sqrt{\varepsilon})$ & $G_{n}=\frac{1}{4 \pi\left|x-x_{j}\right|}+R\left(x ; x_{j}\right)$ & none \\
$O(1)$ & $G_{h}=\frac{\exp \left(-\alpha\left|x-x_{j}\right|\right)}{4 \pi\left|x-x_{j}\right|}+G_{R}\left(x ; x_{j}\right)$ & $\frac{1}{\alpha}$ \\
$O(1 / \varepsilon)$ & $G_{f}=\frac{\exp \left(-\alpha_{2}\left|x-x_{j}\right| / \varepsilon\right)}{4 \pi\left|x-x_{j}\right|}$ & $\frac{\varepsilon}{\alpha_{2}}$ \\
\hline
\end{tabular}

$\lambda \sim R$ or $\alpha=O(1)$. This marks the transition from exponential to algebraic decay and thus from short-range to long-range signaling. Clearly, this transition is independent of the compartment size $\varepsilon$. Here, the decay behavior of the concentration profiles is determined by the Green's function $G_{h}$, which basically decays exponentially to the cell boundary. In the region $\lambda>R$ the mode of decay remains algebraic until $\lambda \sim R / \sqrt{\varepsilon}$ (or $\alpha=O(\sqrt{\varepsilon})$ ), which marks the transition to the constant regime; i.e., for $\lambda \gg R / \sqrt{\varepsilon}$ diffusion is sufficiently fast or the cytosolic deactivation rate so weak that the concentration of activated molecules is basically constant throughout the cytosol. In that regime the decay behavior of the concentration profiles is determined by the algebraic decay of the Neumann function $G_{n}$ in Table 6.1. Note that the particular $\sqrt{\varepsilon}$ dependence of this transition point is the result of our assumption that $\varepsilon \partial_{n x} u=O(1)$ in (2.6c). However, the qualitative conclusion that smaller compartments reach the constant regime for smaller values of $\alpha$ would not be altered if we had, for example, assumed that $\partial_{n x} u=O(1)$.

We derived simple expressions for the local behavior of the concentration profiles in the three different $\alpha$-regimes (cf. (3.20), (3.26), (3.39), and (3.40)). These expressions are similar to the concentration profiles from a point source in infinite space $[16,20]$. Moreover, they extend these earlier results to the case of a linear activation rate (BVP (B) in (2.6c)) and explicitly take into account the order of magnitude of the degradation rate $\alpha$. Depending on the regime, the presence of multiple signaling compartments inside the cell exerts a different influence on the local solutions. In the short-range signaling regimes $\alpha=O(1)$ and $\alpha=O(1 / \varepsilon)$ the local concentration profile is mainly determined by the singular part of the respective Green's function in Table 6.1, while contributions of the other compartments are of $O(\varepsilon)$ or smaller. However, in the long-range signaling regime $\alpha=O(\sqrt{\varepsilon})$ an algebraically decaying gradient builds on top of a constant background level, which depends on the local kinetic enzyme parameters $\kappa_{i}$ of all other compartments.

In our model, the type of signaling gradient is completely determined by the cytosolic deactivation rate $\alpha$ and the size of the intracellular signaling compartment $\varepsilon$. In particular, the local kinetic enzyme parameters $\sigma_{j}$ and $\kappa_{j}$ influence the concentration level of activated signaling molecules only in the neighborhood of the compartments. However, intracellular signaling pathways often involve multiple activation/ deactivation events such that one signaling gradient could, in principle, build on top of another one as in the case of the RanGTP-Importin $\beta$ gradient around mitotic chromosomes [6] or the mitogen-activated protein kinase pathways [12, 14]. Such a mechanism can provide long-range signaling even if the activity of the cytosolic enzyme is high and, as a consequence, the resulting (secondary) signaling gradient will, in general, also depend on the local kinetic enzyme parameters. Recently, it was shown in a one-dimensional model for a signaling cascade mediated by GTPases that 
a particular localization of the antagonistic enzymes in conjunction with feed-forward circuits is sufficient to generate a nonmonotonic signaling gradient at the third level of the cascade [22].

If signaling occurs simultaneously from multiple compartments, the resulting spatial distribution of activated molecules will depend on the particular arrangement of the compartments within the cell. Using our three-dimensional framework, such questions can now be systematically addressed, for example, in the context of the different cell division phases where a significant reorganization of intracellular compartments takes place. This, in turn, requires coordinated "communication" between the compartments where signaling gradients might play a prominent role. Indeed, the formation of gradients of different RanGTP species around mitotic chromosomes is one example in that direction $[6,3]$. Recently, it has been proposed that subcellular signaling gradients might also be important for checkpoint function in metazoan spindle assembly [20]. However, the functional role of centrosomes in the assembly of the bipolar spindle is only partially understood since centrosome-independent spindle formation has been observed in some cell types [25]. In particular, it might be possible that centrosomes are also sources of signaling gradients for which the special antipodal arrangement of these organelles becomes important.

\section{REFERENCES}

[1] COMSOL Multiphysics (v3.4), COMSOL AB, Stockholm, 2007.

[2] G. Barton, Elements of Green's Functions and Propagation: Potentials, Diffusion and Waves, Clarendon Press, Oxford, UK, 1989.

[3] P. Bastiaens, M. Caudron, P. Niethammer, and E. Karsenti, Gradients in the selforganization of the mitotic spindle, Trends Cell. Biol., 16 (2006), pp. 125-134.

[4] P. C. Bressloff, B. A. Earnshaw, and M. J. Ward, Diffusion of protein receptors on a cylindrical dendritic membrane with partially absorbing traps, SIAM J. Appl. Math., 68 (2008), pp. 1223-1246.

[5] G. C. Brown and B. N. Kholodenko, Spatial gradients of cellular phospho-proteins, FEBS Letters, 457 (1999), pp. 452-454.

[6] M. Caudron, G. Bunt, P. Bastiaens, and E. Karsenti, Spatial coordination of spindle assembly by chromosome-mediated signaling gradients, Science, 309 (2005), pp. 1373-1376.

[7] A. M. Ill'In, Matching of Asymptotic Expansions of Solutions of Boundary Value Problems, AMS, Providence, RI, 1992.

[8] K. Jacobson And J. Wojcieszyn, The translational mobility of substances within the cytoplasmic matrix, Proc. Natl. Acad. Sci. USA, 81 (1984), pp. 6747-6751.

[9] L. Jourdain, P. Curmi, A. Sobel, D. Pantaloni, and M.-F. Carlier, Stathmin: A tubulinsequestering protein which forms a ternary $T 2 S$ complex with two tubulin molecules, Biochemistry, 36 (1997), pp. 10817-10821.

[10] P. Kalab, K. Weiss, and R. Heald, Visualization of a Ran-GTP gradient in interphase and mitotic Xenopus egg extracts, Science, 295 (2002), pp. 2452-2456.

[11] J. Kevorkian And J. D. Cole, Multiple Scale and Singular Perturbation Methods, Springer, New York, 1996.

[12] B. N. Kholodenko, Cell-signalling dynamics in time and space, Nat. Rev. Mol. Cell. Biol., 7 (2006), pp. 165-176.

[13] V. S. Kraynov, C. Chamberlain, G. M. Bokoch, M. A. Schwartz, S. Slabaugh, and K. M. HAHN, Localized Rac activation dynamics visualized in living cells, Science, 290 (2000), pp. 333-337.

[14] N. I. Markevich, M. A. Tsyganov, J. B. Hoek, and B. N. Kholodenko, Long-range signaling by phosphoprotein waves arising from bistability in protein kinase cascades, Molec. Systems Biol., 2 (2006), paper 61.

[15] J. Meyers, J. Craig, And D. J. Odde, Potential for control of signaling pathways via cell size and shape, Current Biol., 16 (2006), pp. 1685-1693.

[16] E. NeHER, Concentration profiles of intracellular calcium in the presence of a diffusible chelator, in Exp. Brain Res. 14, Springer, Berlin, Heidelberg, 1986, pp. 80-96.

[17] E. NeHER, Usefulness and limitations of linear approximations to the understanding of $\mathrm{Ca}^{2+}$ signals, Cell Calcium, 24 (1998), pp. 345-357. 
[18] P. Niethammer, P. Bastiaens, and E. Karsenti, Stathmin-tubulin interaction gradients in motile and mitotic cells, Science, 303 (2004), pp. 1862-1866.

[19] S. OzAWA, An asymptotic formula for the eigenvalues of the Laplacian in a three dimensional domain with a small hole, J. Fac. Sci. Univ. Tokyo Sect. IA, 30 (1983), pp. 243-257.

[20] R. P. Sear And M. Howard, Modeling dual pathways for the metazoan spindle assembly checkpoint, Proc. Natl. Acad. Sci. USA, 103 (2006), pp. 16758-16763.

[21] G. D. Smith, L. Dai, R. M. Miura, and A. Sherman, Asymptotic analysis of buffered calcium diffusion near a point source, SIAM J. Appl. Math., 61 (2001), pp. 1816-1838.

[22] J. Stelling and B. Kholodenko, Signaling cascades as cellular devices for spatial computations, J. Math. Biol., 58 (2009), pp. 35-55.

[23] R. Straube, M. J. Ward, and M. Falcke, Reaction rate of small diffusing molecules on a cylindrical membrane, J. Stat. Phys., 129 (2007), pp. 377-405.

[24] R. Thul And M. FALCKe, Release currents of $I P_{3}$ receptor channel clusters and concentration profiles, Biophys. J., 86 (2004), pp. 2660-2673.

[25] H. VARMark, Functional role of centrosomes in spindle assembly and organization, J. Cell. Biochem., 91 (2004), pp. 904-914.

[26] M. J. WARD, Diffusion and bifurcation problems in singularly perturbed domains, Natural Resource Modeling, 13 (2000), pp. 271-302.

[27] M. J. WARD AND J. B. Keller, Strong localized perturbations of eigenvalue problems, SIAM J. Appl. Math., 53 (1993), pp. 770-798.

[28] R. Wollman, E. N. Cytrynbaum, J. T. Jones, T. Meyer, J. M. Scholey, And A. Mogilner, Efficient chromosome capture requires a bias in the 'search-and-capture' process during mitotic-spindle assembly, Current Biol., 15 (2005), pp. 828-832.

[29] H. Yokoe And T. Meyer, Spatial dynamics of GFP-tagged proteins investigated by local fluorescence enhancement, Nat. Biotechnol., 14 (1996), pp. 1252-1256.

Copyright (c) by SIAM. Unauthorized reproduction of this article is prohibited. 